\title{
A hierarchical classification method for finger knuckle print recognition
}

\author{
Tao Kong, Gongping Yang ${ }^{*}$ and Lu Yang
}

\begin{abstract}
Finger knuckle print has recently been seen as an effective biometric technique. In this paper, we propose a hierarchical classification method for finger knuckle print recognition, which is rooted in traditional score-level fusion methods. In the proposed method, we firstly take Gabor feature as the basic feature for finger knuckle print recognition and then a new decision rule is defined based on the predefined threshold. Finally, the minor feature speeded-up robust feature is conducted for these users, who cannot be recognized by the basic feature. Extensive experiments are performed to evaluate the proposed method, and experimental results show that it can achieve a promising performance.
\end{abstract}

Keywords: Biometrics; Finger knuckle print recognition; Hierarchical classification

\section{Introduction}

Recently, a new biometric technology based on finger knuckle print has attracted much attention in the biometrics research community. Finger knuckle print is highly unique, so it can be served as a distinctive biometric identifier [1-5]. Compared with traditional biometric techniques (e.g., faces, fingerprints, and voices), finger knuckle print exhibits some advantages in real application: (1) It is hard to be abraded since people hold stuffs with inner side of their hands. (2) Unlike fingerprint, there is no stigma of criminal investigation associated with finger knuckle print. So, finger knuckle print has a high user acceptance rate [6]. (3) People rarely leave finger knuckle print on stuff surface, making the loss of private data less possible. Hence, finger knuckle print is considered to be one of the most promising biometric techniques for personal identification in future.

Like other biometric techniques, feature extraction and matching play an important role in finger knuckle print recognition. In [1], Zhang et al. used two-dimensional Gabor filter to extract local orientation information, and the angular distance was used to measure the similarity between two competitive coding maps. In [2,7], the bandlimited phase-only correlation (BLPOC)-based method was adopted to match finger knuckle print. Zhang et al.

\footnotetext{
* Correspondence: gpyang@sdu.edu.cn

School of Computer Science and Technology, Shandong University, Jinan 250101, China
}

[3] proposed a method, combining orientation and magnitude features, for finger knuckle print recognition, named ImCompCode\&MagCode. Later, Zhang et al. [4] presented an effective finger knuckle print recognition scheme by extracting and assembling local and global features. In [8], Morales et al. proposed a finger knuckle print authentication system using the scale invariant feature transform (SIFT) exacted from the enhanced image. Further, a finger knuckle print recognition algorithm based on the speeded-up robust features (SURF) was proposed [9]. In [10], local features of the enhanced finger knuckle print image were extracted using SIFT and SURF. Li et al. [11] proposed a finger knuckle print recognition method named the adaptive steerable orientation coding (ASOC). In [12], Zhang and $\mathrm{Li}$ improved the finger knuckle print recognition accuracy using two Riesz transform-based coding schemes, namely RCode1 and RCode2.

In high security applications, the extremely low false accept rate (FAR) and false reject rate (FRR) are desired at the same time, which is called the double-low problem [13]. However, it is difficult to solve this problem only by improving the performance of a certain biometric technique. So, fusion of various techniques becomes a promising way $[4,10,12,14]$. In a multi-biometrics system, fusion can be accomplished by utilizing available information in any of four biometric modules: sensor, feature extraction, matching, and decision modules [15-17]. As matching score contains ample discriminative

\section{穴 Springer}


information, the score-level fusion is fairly popular in the field of biometrics [18].

A number of multi-biometrics systems based on score-level fusion have appeared in recent years. Kittler et al. [19] experimented with several fusion techniques for face and voice, including sum, product, minimum, median, and maximum rules, and they found that sum rule outperforms the others. Ross and Jain [20] combined face, fingerprint, and hand with sum rule, decision tree, and linear discriminant method. About finger knuckle print, Zhang et al. applied a matcher-weighting $(\mathrm{MW})$ rule to fuse local and global features [4]. Later, SIFT and SURF were fused using weighted sum rule [10]. Although multi-biometrics system offers a number of advantages, it is usually more expensive than unibiometric system due to additional hardwares (i.e., computational or storage resources) and larger enrollment and recognition times [15].

In this paper, a hierarchical classification method for finger knuckle print recognition is proposed. Firstly, we take the Gabor feature as the basic feature in the first recognition phase. Then, a new decision rule is defined based on the predefined threshold. Finally, the minor feature SURF is conducted in the second recognition phase for users that are unsuccessfully recognized at the first recognition phase. Extensive experiments are conducted on a database from Hong Kong Polytechnic University (PolyU) [21] to validate the performance of the proposed method. Experimental results show that the proposed method performs well in finger knuckle print recognition.

The rest of this paper is organized as follows: Section 2 introduces the proposed hierarchical classification method. Section 3 reports the experimental results and discussions. Finally, conclusions and future work are presented in Section 4.

\section{The proposed classification method}

\subsection{The classification method}

Traditionally, identity recognition can be regarded as the following two-category classification problem: given a claimed identity $I$ and a query feature set $X^{A}$, we need to decide if $\left(I, X^{A}\right)$ belongs to 'genuine' or 'imposter' class. Let $X_{I}^{E}$ be the enrolled template of identity $I$. Typically, a matching score $s$, which measures the similarity or dissimilarity between $X^{A}$ and $X_{I}^{E}$, is computed. If $s$ measures dissimilarity, the decision rule is shown in Equation 1:

$$
\left(I, X^{A}\right)=\left\{\begin{array}{l}
\text { Genuine if }(s<\delta) \\
\text { Imposter if }(s \geq \delta)
\end{array}\right.
$$

where $\delta$ is a predefined threshold. Figure 1 shows the matching results of this decision rule.

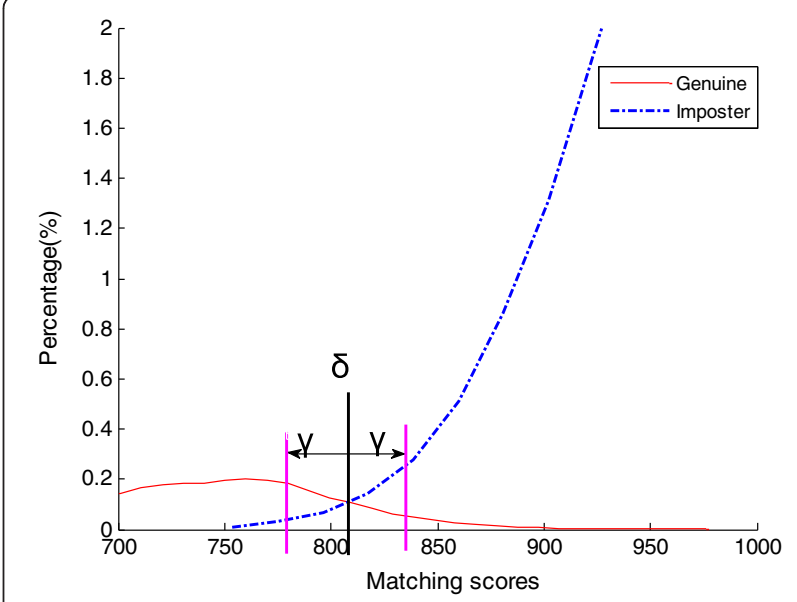

Figure 1 Matching score distributions near the threshold $\delta$.

In Figure 1, the scores near the threshold $\delta$ contribute a large part of FRR and FAR. For example, genuine users with matching scores in $[\delta, \delta+\gamma]$ will be rejected falsely, which will lead to FRR. Similarly, imposter users with matching scores in $[\delta-\gamma, \delta]$ will be accepted falsely, which will result in FAR. So, we propose a new method to improve the classification rule.

The proposed hierarchical classification method consists of two phases. In the first phase, the basic feature is extracted to conduct recognition. To judge whether recognition is successful, a decision rule is given. If the matching criterion is met, then the recognition process comes to an end. Otherwise, the second phase is performed using the minor feature, and a recognition result will be given. The framework of the proposed method is shown in Figure 2.

Given a predefined threshold $\delta$, the proposed decision rule is shown in Equation 2:

$$
\left(I, X^{A}\right)=\left\{\begin{array}{l}
\text { Genuine if }(s \leq \mathrm{v} \delta-\gamma) \\
\text { Imposter if }(s \geq \delta+\gamma) \\
\text { Undecided if }(\delta-\gamma<s<\delta+\gamma)
\end{array}\right.
$$

where $\gamma$ is a small perturbance for the threshold $\delta$. In Equation 2, if the matching score $s \leq \delta-\gamma$, the claimed identity will be accepted as 'genuine'. Similarly, if the score $s \geq \delta+\gamma$, the claimed identity will be rejected as 'imposter'. In these cases, by the basic feature, we can be completely sure whether the user is genuine or imposter, and the recognition process is over. But, if the score $s$ is between $\delta-\gamma$ and $\delta+\gamma$, we cannot recognize the user only using the basic feature. In this case, we need to further extract the minor feature for the second matching phase. The traditional decision rule, as shown in Equation 1, will be used to give the recognition result.

In this paper, we separately use Gabor [22] and SURF [23] to extract the basic and minor features. The Gabor 


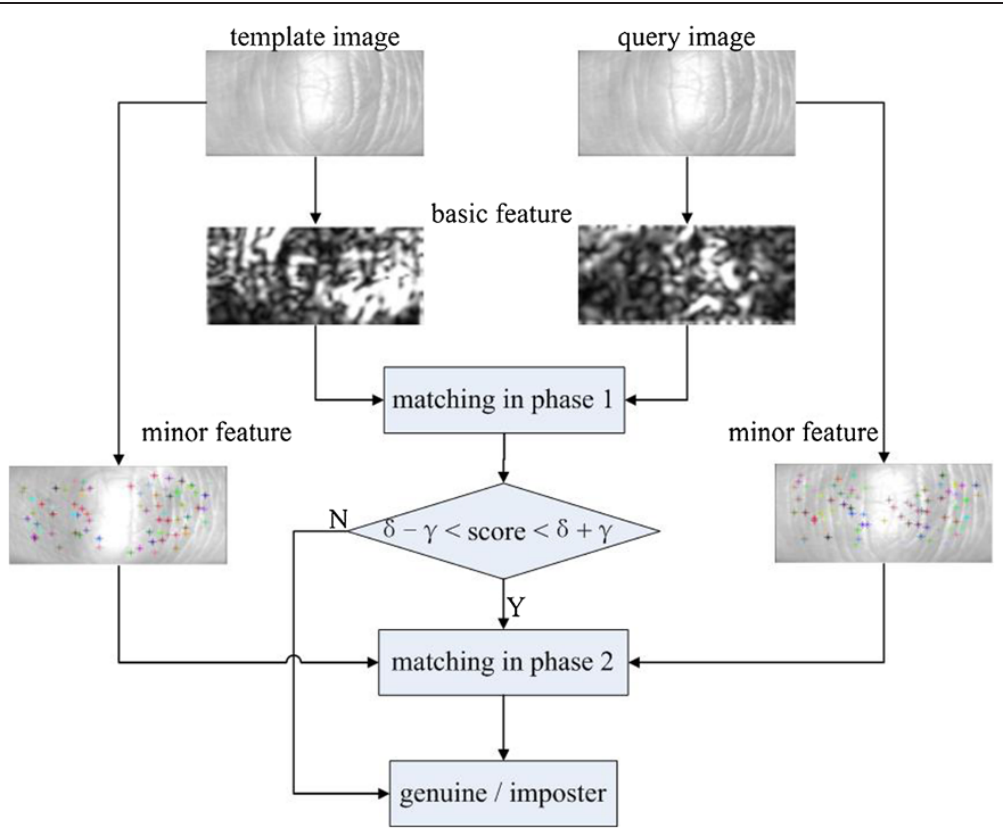

Figure $\mathbf{2}$ The schematic diagram of the hierarchical classification method.

feature can efficiently reflect the texture of an image, and SURF emphasizes key points in an image, such as corner and extreme points. Hence, better recognition accuracy can be achieved if they can be appropriately combined. The fundamental idea of the proposed method is to deliver the recognition work to SURF when Gabor cannot perform recognition successfully. By supplement of SURF feature to Gabor feature, we can improve recognition performance. Although we show the proposed classification method in the case of Gabor and SURF, it can be easily generalized by two other features with complementarity and even two other biometric techniques.

\subsection{Matching}

During recognition, the Gabor feature is firstly used, and matching scores are computed using the nearestneighbor-ratio method [24]. Let $G_{\mathrm{q}}=\left\{g_{1}^{(\mathrm{q})}, g_{2}{ }^{(\mathrm{q})}, \cdots\right.$, $\left.g_{\mathrm{d}}^{(\mathrm{q})}\right\}$ and $G_{\mathrm{e}}=\left\{g_{1}^{(\mathrm{e})}, g_{2}^{(\mathrm{e})}, \cdots, g_{\mathrm{d}}^{(\mathrm{e})}\right\}$ be Gabor feature vectors of query and enrolled images, respectively, and the distance between $G_{i}$ and $G_{j}$ is defined by Equation 3:

$$
d\left(G_{\mathrm{q}}, G_{\mathrm{e}}\right)=\sum_{k=1}^{d}\left\|g_{k}^{(\mathrm{q})}-g_{k}^{(\mathrm{e})}\right\|_{2}
$$

where $\left\|g_{k}^{(\mathrm{q})}-g_{k}^{(\mathrm{e})}\right\|_{2}$ denotes the Euclidean distance between $g_{k}^{(i)}$ and $g_{k}^{(j)}$.

And then let $S_{\mathrm{q}}=\left\{s_{1}{ }^{(\mathrm{q})}, s_{2}{ }^{(\mathrm{q})}, \cdots, s_{\mathrm{m}}{ }^{(\mathrm{q})}\right\}$ and $S_{\mathrm{e}}=\left\{s_{1}{ }^{(\mathrm{e})}\right.$, $\left.s_{2}{ }^{(\mathrm{e})}, \cdots, s_{\mathrm{n}}{ }^{(\mathrm{e})}\right\}$ be SURF feature vectors of the query and enrolled images, respectively, and $s_{i}{ }^{(\mathrm{q})}$ and $s_{i}{ }^{(\mathrm{e})}$ are feature vectors of key point $i$ in $S_{\mathrm{q}}$ and key point $j$ in $S_{\mathrm{e}}$ separately. The matching rule [10] is defined as follows:

$$
s_{i}^{(\mathrm{q})}=\left\{\begin{array}{l}
\text { Matched with } \mathrm{s}_{j}^{(\mathrm{e})} \text { if } \frac{\left\|s_{i}^{(\mathrm{q})}-s_{j}^{(\mathrm{e})}\right\|_{2}}{\left\|s_{i}^{(\mathrm{q})}-s_{k}^{(\mathrm{e})}\right\|_{2}}<T \\
\text { Unmatched otherwise }
\end{array},\right.
$$

where $\left\|s_{i}^{(\mathrm{q})}-s_{j}^{(\mathrm{e})}\right\|_{2}$ is the Euclidean distance between $s_{i}^{(\mathrm{q})}$ and its first nearest neighbor $s_{j}^{(\mathrm{e})}$, and $\left\|s_{i}^{(\mathrm{q})}-s_{k}^{(\mathrm{e})}\right\|_{2}$ denotes the distance between $s_{i}^{(\mathrm{q})}$ and its second nearest neighbor $s_{k}^{(\mathrm{e})}$. Besides, $T$ is a predefined threshold.

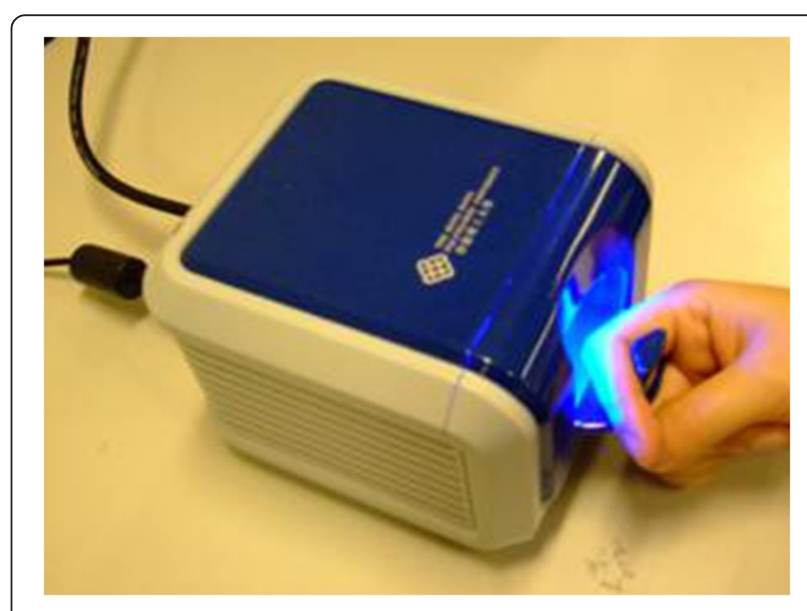

Figure 3 The device used to collect finger knuckle print images. 


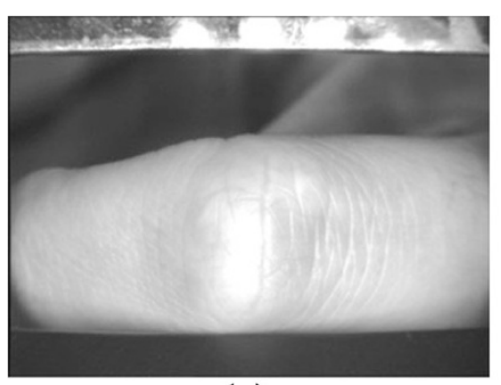

(a)

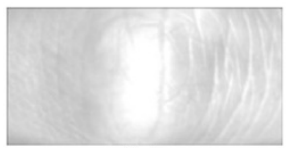

(c)

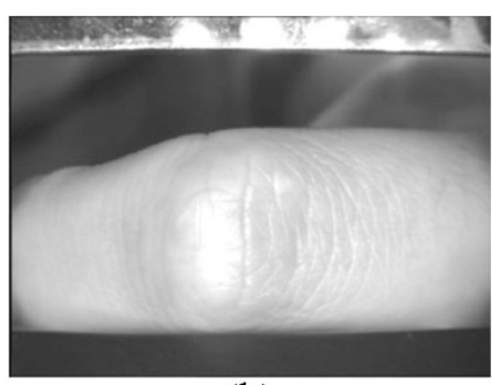

(b)

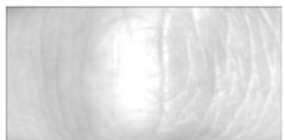

(d)

Figure 4 Some typical images. (a,b) Original finger knuckle print images. (c,d) Corresponding ROI images.

\section{Experimental results and analyses}

\subsection{Database and benchmarks}

We use the PolyU finger knuckle print database [21] to evaluate the performance of the proposed method. This database is collected from 165 volunteers, including 125 males and 40 females. These images are collected in two separate sessions. In each session, the subject is asked to provide six images for the left index and middle fingers, and the right index and middle fingers. In total, the database contains 7,920 images with 12 images per finger. As images of a finger belong to a class, there are 660 classes. Figure 3 shows the image acquisition device. The database provides two sets of images. There are alloriginal images in the first set, and the corresponding ROI images, with size of $110 \times 220$ pixels, are in another set. Some typical images are presented in Figure 4.

Experiments are performed both in verification and identification modes. In the verification mode, the performance of the proposed method is evaluated by the

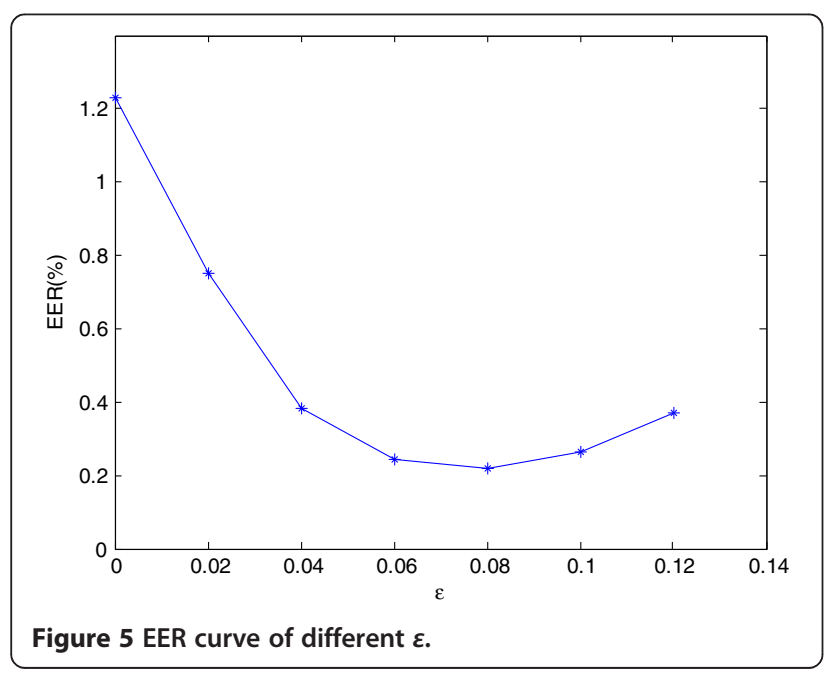

equal error rate (EER). The EER is the error rate when FRR is equal to FAR, which can reflect the overall performance of a biometric system [25]. And, we also generate the receiver operating characteristic (ROC) curves of the different methods in this mode. In the identification mode, we use the cumulative match curves (CMC), and rank-one recognition rate as a benchmark.

\subsection{Threshold determination}

In this section, we discuss the influence of parameter settings on the performance of the proposed method and get the best parameter value. As shown in Equation 2 , our method's performance is affected by two parameters: $\delta$ and $\gamma$. Parameter $\delta$ can be defined as the corresponding matching score when FAR is equal to FRR, and parameter $\gamma$ determines the number of users that Gabor cannot recognize successfully. So, the experimental performance will only be affected by the parameter $\gamma$.

Let $\gamma=\delta \times \varepsilon$, and changing $\varepsilon$ will lead to variation of $\gamma$. We will test the impact of parameter $\varepsilon$ on the entire database. Figure 5 shows the EER curve generated by different values of $\varepsilon$, and Table 1 lists the corresponding EER values.

\section{Table 1 EERs (\%) of different $\varepsilon$}

\begin{tabular}{ll}
\hline $\boldsymbol{\varepsilon}$ & EER \\
\hline 0 & 1.230 \\
0.02 & 0.753 \\
0.04 & 0.382 \\
0.06 & 0.244 \\
0.08 & 0.218 \\
0.10 & 0.265 \\
0.12 & 0.369 \\
\hline
\end{tabular}


From the experimental results, we can see that when $\varepsilon=0.08$, the EER reaches the minimum value of $0.218 \%$. As the recognition ability of the minor feature SURF is limited, the recognition performance will decrease when $\varepsilon$ is larger than 0.08 . Besides, the larger $\varepsilon$, the longer the recognition time. Therefore, we claim that when $\varepsilon=$ 0.08 , the system achieves an optimal performance, with the EER of $0.218 \%$. In the following experiments, we determine $\gamma$ by $\varepsilon$.

\subsection{Experiments}

In this paper, all experiments are implemented in Matlab 2011a on a PC with 3.10-GHz CPU and 2.0-GB memory. Five experiments are designed to evaluate the performance of the proposed method. Experiment 1 evaluates the performance of the proposed method in verification mode. Experiment 2 evaluates the performance of the proposed method on different fingers. Experiment 3 is conducted in identification mode. Experiment 4 measures the processing time. Finally, the influence of the feature order in fusion is discussed in experiment 5 .

In the experiments, we compare the proposed method with four traditional score-level fusion methods, namely simple sum (SS), min score (MIN), max score (MAX), and matcher weighting (MW). Gabor and SURF are, respectively, used to extract the basic and minor features in the proposed method. Besides, the images collected in the first session will be used as the gallery set, and the others will be used as the probe set.

\subsubsection{Experiment 1: verification experiment on all fingers}

In this experiment, all images are involved. Each probe image is matched with all gallery images. Therefore, there are 23,760 intraclass matchings and 15,657,840 interclass matchings. Parameters are set as follows: $\delta=$ 868.8 and $\gamma=69.5$. Experimental results of the various fusion methods are summarized in Table 2, and the corresponding ROC curves are illustrated in Figure 6.

It can be ascertained from Table 2 and Figure 6 that the proposed method achieves the best performance among all the methods considered in this work. These traditional methods (i.e., SS, MIN, and MAX) fuse basic

Table 2 EERs (\%) of different methods

\begin{tabular}{ll}
\hline Method & EER \\
\hline Gabor & 1.23 \\
SURF & 8.20 \\
SS (Gabor + SURF) & 2.48 \\
MIN (Gabor + SURF) & 3.25 \\
MAX (Gabor + SURF) & 4.13 \\
MW (Gabor + SURF) & 0.31 \\
Proposed & 0.22 \\
\hline
\end{tabular}

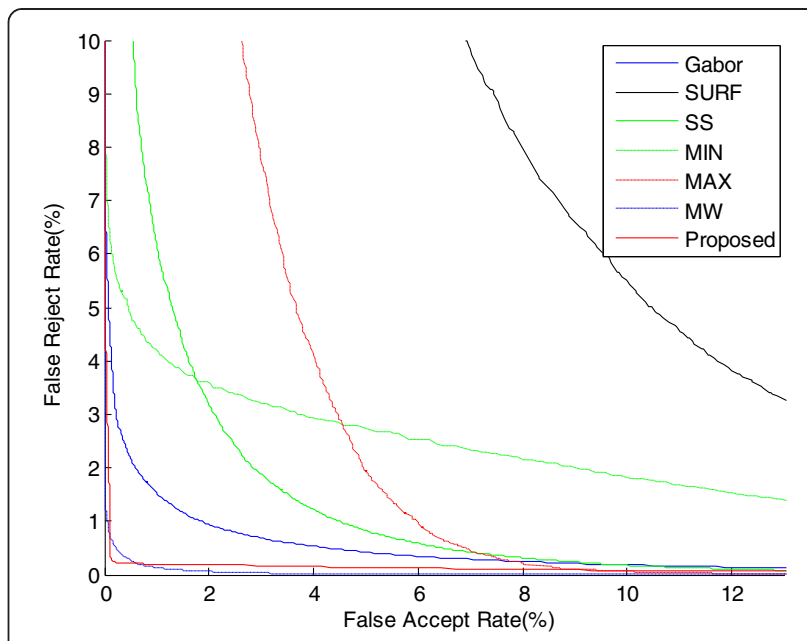

Figure 6 ROC curves of the different methods.

and minor features for all users. So, the recognition performance of two features has not been fully explored. Meanwhile, the matching score distributions of two features may be very different. These two reasons lead to the inferior performance of traditional fusion methods. The matcher-weighting fusion method takes into account different recognition performances of features; thus, it makes effective improvement of performance. But, it still uses all matching scores for fusion. In short, the recognition performance of the proposed method is the best.

We also compare the proposed method with other existing finger knuckle print recognition systems. All these systems have performed on the PolyU finger knuckle print database, and fused two or more features for recognition. Table 3 shows the EERs of these systems. It can be observed that only the EER of Enh-FUSE [10] is lower than that of our method. However, as the performance of Enh-FUSE depends on SIFT and SURF features, and the extraction of these features are quite time consuming; Enh-FUSE works much slower than the proposed method. In one word, the proposed method can achieve a promising balance between verification accuracy and verification speed.

Table 3 EER (\%) of the proposed method and other methods proposed in $[3,4,10,26]$

\begin{tabular}{lc}
\hline Method & EER \\
\hline ImCompCode\&MagCode [3] & 1.48 \\
LFI [26] & 1.27 \\
LGIC [4] & 0.402 \\
LGIC2 [4] & 0.358 \\
Enh-FUSE [10] & 0.215 \\
Proposed & 0.218 \\
\hline
\end{tabular}




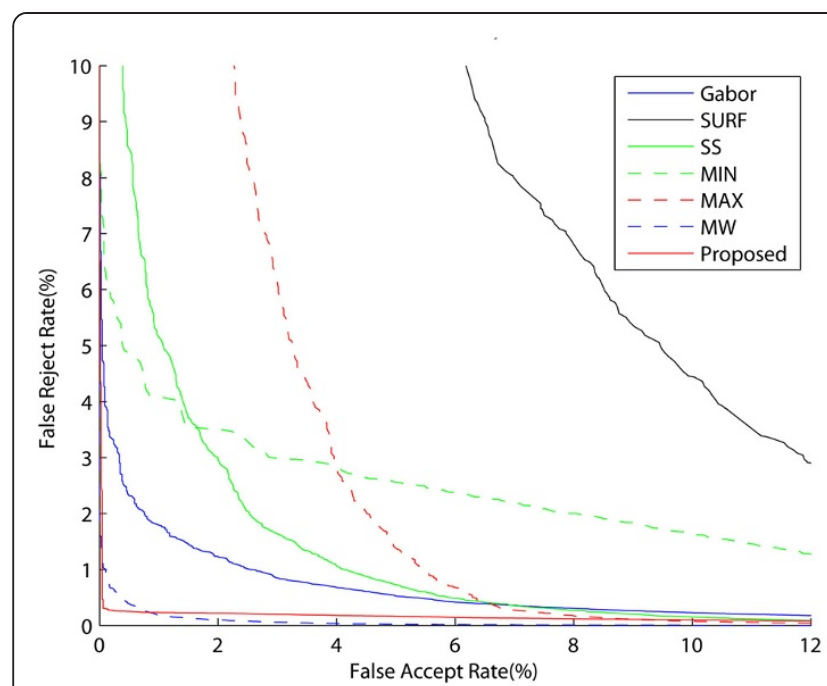

(a)

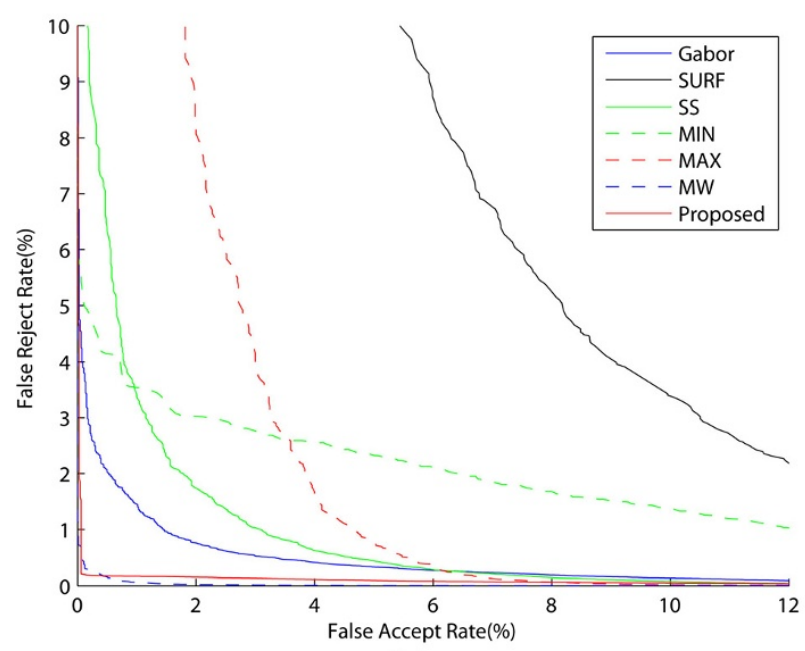

(c)

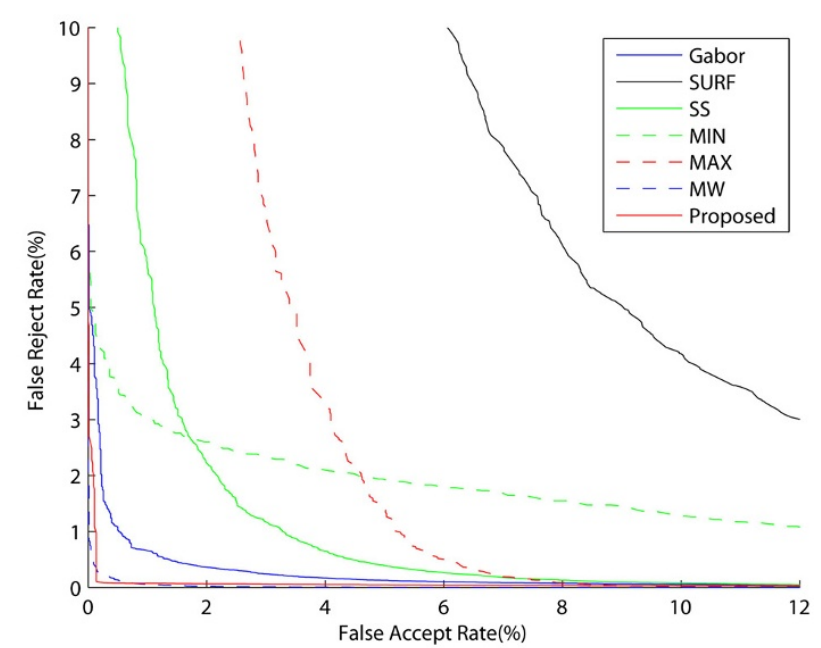

(b)

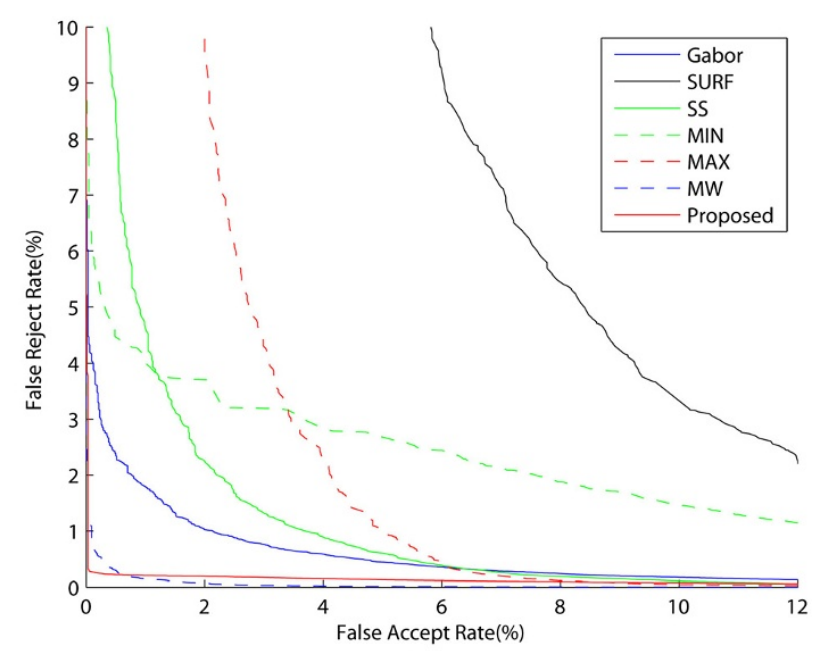

(d)

Figure 7 ROC curves of the different fingers. (a) Left index fingers, (b) left middle fingers, (c) right index fingers, (d) right middle fingers.

\subsubsection{Experiment 2: verification experiment on each type of finger}

As mentioned in Section 3.1, the open database contains finger knuckle prints from four types of fingers. The aim of this experiment is to evaluate the performance of the proposed method on each type of finger. For each type of finger, there are 5,940 intraclass matchings and 974,160 interclass matchings. And, four groups of parameters are set as follows: $\delta_{1}=862.5, \gamma_{1}=69.0 ; \delta_{2}=$ $874.9, \gamma_{2}=70.0 ; \delta_{3}=871.0, \gamma_{3}=69.7 ;$ and $\delta_{4}=870.4, \gamma_{4}=$ 69.6. The ROC curves are shown in Figure 7 , and the EERs are listed in Table 4.

From Figure 7 and Table 4, we can see that the proposed method achieves much lower EER than the traditional score-level fusion methods in each type of finger.
Table 4 EERs (\%) of the different fingers

\begin{tabular}{lcccc}
\hline Method & $\begin{array}{c}\text { Left } \\
\text { index } \\
\text { fingers }\end{array}$ & $\begin{array}{c}\text { Left } \\
\text { middle } \\
\text { fingers }\end{array}$ & $\begin{array}{c}\text { Right } \\
\text { index } \\
\text { fingers }\end{array}$ & $\begin{array}{c}\text { Right } \\
\text { middle } \\
\text { fingers }\end{array}$ \\
\hline Gabor & 1.47 & 0.74 & 1.23 & 1.42 \\
SURF & 7.50 & 7.31 & 6.87 & 7.04 \\
SS (Gabor + SURF) & 2.33 & 2.09 & 1.89 & 2.12 \\
MIN (Gabor + SURF) & 3.04 & 2.45 & 2.83 & 3.20 \\
MAX (Gabor + SURF) & 3.72 & 3.70 & 3.24 & 3.17 \\
MW (Gabor + SURF) & 0.44 & 0.23 & 0.24 & 0.37 \\
ImCompCode\&Magcode [3] & 1.73 & 1.78 & 1.44 & 1.64 \\
Proposed & 0.26 & 0.15 & 0.20 & 0.24 \\
\hline
\end{tabular}




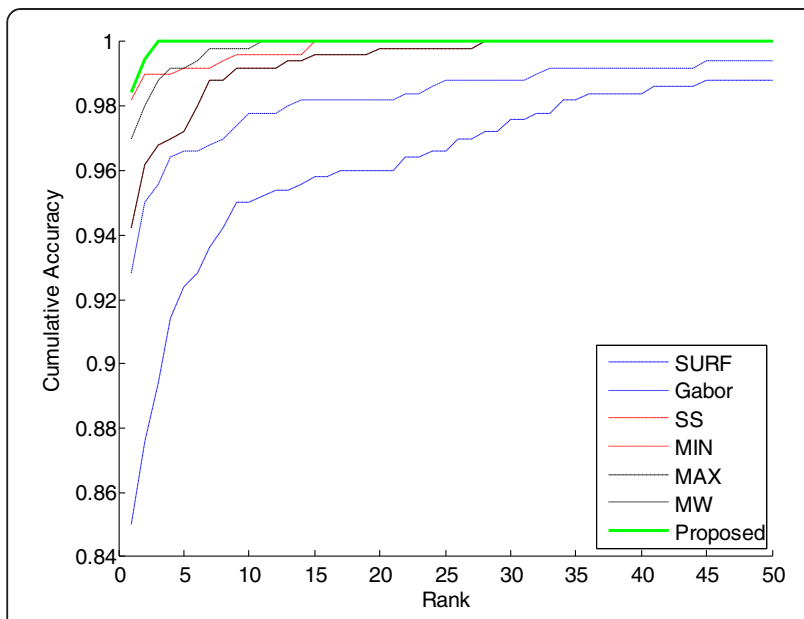

Figure 8 Cumulative match curves of the different methods.

Specially, the left middle fingers perform better than the other fingers with the lowest EER of $0.15 \%$.

\subsubsection{Experiment 3: identification experiment on all fingers}

In this experiment, we evaluate the performance of the proposed method in identification mode, in which we choose the first image in each class as gallery sample and other 11 ones as probe samples. Therefore, we get 660 gallery samples and 7,260 $(660 \times 11)$ probe samples totally. The CMCs are shown in Figure 8. The rank-one recognition rates are listed in Table 5. From the experimental results, we can see that the proposed method is better than the traditional score-level fusion methods in the identification mode. The rank-one rate for the proposed method is $98.5 \%$, which is the highest among all methods.

\subsubsection{Experiment 4: experiment about processing time}

In this experiment, the average processing time of all the images is measured, as shown in Table 6. In this experiment $\delta=868.8$ and $\gamma=69.5$. The total processing time of one image is less than $0.2 \mathrm{~s}$, which is fast enough for real-time applications.

Table 5 Rank-one recognition rate (\%) of different methods

\begin{tabular}{lc}
\hline Method & Rank-one recognition rate \\
\hline Gabor & 85.0 \\
SURF & 92.8 \\
SS (Gabor + SURF) & 98.2 \\
MIN (Gabor + SURF) & 94.2 \\
MAX (Gabor + SURF) & 94.2 \\
MW (Gabor + SURF) & 97.0 \\
Proposed & 98.5 \\
\hline
\end{tabular}

Table 6 The average processing time (ms)

\begin{tabular}{lccc}
\hline Process & Gabor & SURF & Total \\
\hline Feature extraction & 166.42 & 1.92 & 168.34 \\
Matching & 6.81 & 0.08 & 6.89 \\
Total & 173.23 & 2.00 & 175.23 \\
\hline
\end{tabular}

3.3.5 Experiment 5: experiment about the order of features In the proposed method, we take Gabor as the basic feature and SURF as the minor feature. In order to discuss the influence of the feature order in fusion, we reversely take SURF as the basic feature and Gabor as the minor feature. For four types of fingers, four groups of parameters are set as $\delta_{1}=0.692, \gamma_{1}=0.083 ; \delta_{2}=0.625, \gamma_{2}=$ $0.087 ; \delta_{3}=0.691, \gamma_{3}=0.082 ;$ and $\delta_{4}=0.692, \gamma_{4}=0.097$. For all fingers, the parameters are set as $\delta=0.671$ and $\gamma=$ 0.094. The EERs are listed in Table 7.

From Table 7, we can see that no matter which feature is used as the basic feature, the fusion method achieves better recognition performance compared with the basic features. At the same time, the feature order in fusion has a certain influence on the performance. The EER of Gabor + SURF is $0.218 \%$; however; that of SURF + Gabor is $3.20 \%$. It indicates that the features used to fusion need to meet one condition: the basic feature should have a good-enough recognition performance. Gabor + SURF can meet this condition, so it can get a good recognition performance. If we choose SURF, whose recognition performance is limited, as the basic feature, the performance will be not the best. Besides, processing times will be different when the basic feature varies. In our experiments, the processing time of SURF + Gabor is $220.24 \mathrm{~ms}$, which is higher than that of Gabor + SURF. In conclusion, the Gabor + SURF will achieve better recognition performance.

\subsection{Discussion}

Five experiments are performed to evaluate the performance of the proposed method. Experimental results verify that the proposed method can achieve satisfactory recognition in real time. However, the proposed hierarchical classification method has some limitations. Like traditional fusion methods, the features used in the proposed hierarchical fusion method should be complementary to

Table 7 EERs (\%) on the different fingers

\begin{tabular}{lccccc}
\hline Method & $\begin{array}{c}\text { Left } \\
\text { index } \\
\text { fingers }\end{array}$ & $\begin{array}{c}\text { Left } \\
\text { middle } \\
\text { fingers }\end{array}$ & $\begin{array}{c}\text { Right } \\
\text { index } \\
\text { fingers }\end{array}$ & $\begin{array}{c}\text { Right } \\
\text { middle } \\
\text { fingers }\end{array}$ & $\begin{array}{c}\text { All } \\
\text { fingers }\end{array}$ \\
\hline Gabor & 1.47 & 0.74 & 1.23 & 1.42 & 1.23 \\
SURF & 7.50 & 7.31 & 6.87 & 7.04 & 8.20 \\
Gabor + SURF & 0.26 & 0.15 & 0.20 & 0.24 & 0.218 \\
SURF + Gabor & 3.14 & 3.10 & 3.23 & 2.40 & 3.20 \\
\hline
\end{tabular}


some extent, and the performance of the proposed method will be affected by the setting of the parameter. If two features are not complementary, fusion by any method is meaningless. And, there are two parameters: $\delta$ and $\gamma$ in the proposed method. The value of parameter $\delta$ is constant, which is the corresponding matching score of the basic feature when FAR is equal to FRR. It means that only parameter $\gamma$ can affect the performance of the proposed method, and it is set as an empirical value to obtain the best recognition performance.

In addition, the proposed method has one underlying requirement for the basic feature: it should have a goodenough recognition performance. For two features, different feature orders in fusion will result in different recognition performances. The main reason is that the basic feature plays an important role in the proposed method, which should meet the above requirement. If the basic feature has poor recognition performance, it will be very hard to achieve a promising performance. The proposed method has only tested on Gabor and SURF features. Whether other features are effective depends on whether the basic and minor features complement each other. Further tests are required for using other features.

\section{Conclusion and future work}

A hierarchical classification method of finger knuckle print recognition is proposed in this paper. The proposed method consists of two phases. In the first phase, recognition based on the basic feature is conducted. To judge whether matching is successful, the successful matching criteria are given. The second phase is conducted using the minor feature only when the basic feature cannot perform recognition successfully. The proposed system has been evaluated on the public PolyU finger knuckle print database. It is observed that the proposed method can actually achieve a desired balance between verification accuracy and verification speed. The future work will focus on applying the proposed method on other biometrics such as finger vein and face.

\section{Competing interests}

The authors declare that they have no competing interests.

\begin{abstract}
Acknowledgements
The work is supported by the National Natural Science Foundation of China under Grant No. 61173069 and Shandong Natural Science Funds for Distinguished Young Scholar under Grant No. JQ201316. In addition, the authors would particularly like to thank the anonymous reviewers for their helpful suggestions.
\end{abstract}

Received: 18 July 2013 Accepted: 21 March 2014

Published: 2 April 2014

\section{References}

1. L Zhang, L Zhang, D Zhang, Finger-knuckle-print: a new biometric identifier. Paper presented at the 16th IEEE international conference on image processing, (Cairo, Egypt). 7-10 Nov 2009
2. L Zhang, L Zhang, D Zhang, Finger-knuckle-print verification based on band-limited phase-only correlation. Paper presented at the 13th international conference on computer analysis of images and patterns, (Münster, Germany). 2-4 Sept 2009

3. L Zhang, L Zhang, D Zhang, $\mathrm{H}$ Zhu, Online finger-knuckle-print verification for personal authentication. Patt. Recogn. 43, 2560-2571 (2010)

4. L Zhang, L Zhang, D Zhang, $\mathrm{H}$ Zhu, Ensemble of local and global information for finger-knuckle-print recognition. Patt. Recogn. 44, 1990-1998 (2011)

5. KK Sricharan, AA Reddy, A Ramakrishnan, Knuckle based hand correlation for user authentication. Paper presented at the international Society for Optics and Photonic, (California, USA). 13-17 Aug 2006

6. A Kumar, C Ravikanth, Personal authentication using finger knuckle surface. IEEE Trans. Inf. Forensics Security 4, 98-110 (2009)

7. S Aoyama, K Ito, T Aok, Finger-knuckle-print recognition using BLPOC-based local block matching. Paper presented at the first Asian conference on pattern recognition, (Beijing, China). 28-30 Nov 2011

8. A Morales, C Travieso, M Ferrer, J Alonso, Improved finger-knuckle-print authentication based on orientation enhancement. Electron. Lett. 47, 380-381 (2011)

9. Z Le-qing, Finger knuckle print recognition based on surf algorithm. Paper presented at the 8th international conference on fuzzy systems and knowledge discovery, (Shanghai, China). 26-28 July 2011

10. G Badrinath, A Nigam, P Gupta, An efficient finger-knuckle-print based recognition system fusing sift and surf matching scores. Paper presented at the 13th international conference on information and communications security, (Beijing, China). 23-26 Nov 2011

11. Z Li, K Wang, W Zuo, Finger-knuckle-print recognition using local orientation feature based on steerable filter. Paper presented at the 8th international conference on emerging intelligent computing technology and applications, (Huangshan, China). 25-29 July 2012

12. L Zhang, H Li, Encoding local image patterns using Riesz transforms: with applications to palm print and finger-knuckle-print recognition. Image Vision Comput. 30, 1043-1051 (2012)

13. $Y$ Yin, $Y$ Ning, $Z$ Yang, A hybrid fusion method of fingerprint identification for high security applications. Paper presented at the 17th international conference on image processing, (Hong Kong, China). 26-29 Sept 2010

14. K Nandakumar, Y Chen, SC Dass, AK Jain, Likelihood ratio-based biometric score fusion. IEEE Trans. Pattern Anal. Mach. Intell. 30, 342-347 (2008)

15. AA Ross, K Nandakumar, AK Jain, Handbook of Multibiometrics (Springer Berlin, 2006)

16. AK Jain, A Ross, S Prabhakar, An introduction to biometric recognition. IEEE Trans. Circuits Syst. Video Technol. 14, 4-20 (2004)

17. Y Yang, G Yang, $S$ Wang, Finger vein recognition based on multi-instance. Int. J. Digital Content Tech. Appl. 6, 86-94 (2012)

18. M Hanmandlu, J Grover, A Gureja, H Gupta, Score level fusion of multimodal biometrics using triangular norms. Pattern Recognit. Lett. 32, 1843-1850 (2011)

19. J Kittler, M Hatef, RPW Duin, J Matas, On combining classifiers. IEEE Trans. Pattern Anal. Mach. Intell. 20, 226-239 (1998)

20. A Ross, A Jain, Information fusion in biometrics. Pattern Recognit. Lett. 24, 2115-2125 (2003)

21. PolyU, FKP Database, (The Hong Kong Polytechnic University, Hong Kong, 2010). http://www4.comp.polyu.edu.hk/ biometrics/FKP.htm. Accessed 21 Sept 2012

22. C Liu, H Wechsler, Gabor feature based classification using the enhanced fisher linear discriminant model for face recognition. IEEE Trans. Image Process. 11, 467-476 (2002)

23. H Bay, T Tuytelaars, L Van Gool, Surf: speeded up robust features. Paper presented at the 9th European conference on computer vision, (Graz, Austria). 7-13 May 2006

24. K Mikolajczyk, C Schmid, A performance evaluation of local descriptors. IEEE Trans. Pattern Anal. Mach. Intell. 27, 1615-1630 (2005)

25. $G$ Yang, $X X i, Y$ Yin, Finger vein recognition based on a personalized best bit map. Sensors 12, 1738-1757 (2012)

26. L Zhang, L Zhang, D Zhang, Z Guo, Phase congruency induced local features for finger-knuckle-print recognition. Patt. Recogn. 45, 2522-2531 (2012)

doi:10.1186/1687-6180-2014-44

Cite this article as: Kong et al:: A hierarchical classification method for finger knuckle print recognition. EURASIP Journal on Advances in Signal Processing 2014 2014:44. 